Biologie Aujourd'hui, 205 (4), 235-243 (2011)

(C) Société de Biologie, 2012

DOI: $10.1051 / \mathrm{jbio} / 2011019$

\title{
Cryoconservation de l'ovaire : modèles expérimentaux de réimplantation
}

\author{
Nadine Binart ${ }^{1,2}$ et Frédérique Sauvat ${ }^{3}$ \\ 1 Inserm, U693, 94276 Le Kremlin Bicêtre, France \\ ${ }^{2}$ Université Paris-Sud, Faculté de Médecine Paris-Sud, UMR-S693, 94276 Le Kremlin-Bicêtre, France \\ 3 Service de Chirurgie Infantile, CHR Félix Guyon, 97405 Saint Denis, France
}

Auteur correspondant : Nadine Binart, nadine.binart@inserm.fr

Reçu le 13 juillet 2011

Résumé - Les tumeurs malignes chez l'enfant représentent $1 \%$ de tous les cancers. Or, ces enfants et adolescents survivent dans plus de $75 \%$ des cas au prix de séquelles en termes de fertilité avec une destruction quasi constante de la fonction ovarienne chez les filles. Ce risque est maximal chez les patientes traitées par des chimiothérapies à haute dose et/ou comportant des alkylants, par une radiothérapie ou recevant un conditionnement avant une greffe de cellules souches hématopoïétiques. Plusieurs options ont été développées pour préserver la fonction ovarienne: une approche médicamenteuse, une approche chirurgicale et des techniques d'assistance médicale à la procréation. Parmi celles-ci, la congélation embryonnaire est la technique de choix en termes de succès mais elle est limitée aux femmes en âge de procréer ; la cryoconservation ovocytaire mature nécessite une hyper-stimulation ovarienne et le délai nécessaire à ce recueil est souvent incompatible avec l'urgence du traitement anti-tumoral. Ces deux techniques de congélation sont impossibles chez des enfants non pubères et donc la congélation de cortex ovarien reste la seule option car elle permet la conservation de cellules immatures. Le but de l'étude a été d'évaluer les possibilités de restauration de la fonction endocrine (puberté, fonction hormonale cyclique) et de la fertilité après réimplantation d'ovaires prélevés avant la puberté dans deux modèles animaux, la souris en raison de sa prolificité et la brebis à cause de sa physiologie ovarienne proche de celle de la femme. Les résultats démontrent qu'il est possible de restaurer une puberté et une fertilité dans les deux modèles. Cependant, les risques liés à ces traitements ne peuvent être négligés, en particulier les anomalies épigénétiques pour la descendance. Les résultats renforcent la légitimité de proposer une congélation ovarienne chez les filles non pubères qui doivent recevoir un traitement potentiellement stérilisant, pour restaurer leur fertilité, comme cela est proposé chez les femmes adultes. L'information donnée aux patientes et à leurs parents au moment du prélèvement ovarien doit rester prudente quant à l'instauration d'une puberté spontanée, du maintien de la fonction endocrinienne à long terme et du risque malformatif pour leurs futurs enfants.

Mots clés : Ovaire / cryoconservation / greffe / marques épigénétiques

Abstract - Cryopreserved ovaries: models of experimental grafting.

Childhood cancers account for $1 \%$ of malignant tumors. As a result of advances in treatment, almost $80 \%$ of children and adolescents who currently receive a diagnosis of cancer become long-term survivors. Assessment of potential for fertility preservation should thus be a systematic element of care for children treated for a malignant tumor (high-dose chemotherapy with alkylizing agents, radiation therapy including the gonads) or those receiving hematopoietic stem cell grafts for malignant or benign disease (sicklecell anemia, immune deficit). Potential adverse consequences of treatment include impaired puberty and fertility due to gonadal removal, genital tract injury or damage to germ cells from adjuvant therapy. Advances in assisted reproductive technologies have led to new 
possibilities for the prevention and treatment of infertility. Among them, cryopreservation of ovarian tissue appears to be the most promising, or perhaps the only one available before puberty with encouraging results. Nevertheless the uncertainties, or even risks, related to these treatments, should not be neglected. We review experimental data in mouse and sheep animal models. The results demonstrate that immature ovarian grafting can restore spontaneous puberty and fertility in both models. This study addresses the very important issue of epigenetics, and provides valuable information for the study of ovarian transplantation, suggesting that these procedures do not perturb normal epigenetic marks. These results are highly relevant to the question of immature cortex reimplantation.

Key words: Ovary / cryopreservation / grafting / epigenetic marks

\section{Introduction}

L'incidence des tumeurs malignes de l'enfant représente $1 \%$ de tous les cancers et est de l'ordre de 122 cas par million d'enfants (Skinner et al., 2006), taux identique dans la plupart des pays industrialisés. En France, on dénombre 1800 cas de nouveaux patients d'âge pédiatrique par an, dont 700 adolescents âgés de 15 à 19 ans (Stiller et al., 2006) et aux États-Unis, 7500 nouveaux cas pédiatriques par an recensés en 1990.

Les progrès pharmacologiques (chimiothérapie à haute dose, greffe de cellules souches hématopoïétiques...) et la meilleure compréhension des processus de tumorigenèse ont permis d'améliorer de façon notable la survie des jeunes patients traités pour une tumeur maligne, avec un taux de survie globale qui est passé de moins de $50 \%$ en 1973 à plus de $75 \%$ aujourd'hui. Cela signifie qu'en 2010, entre un adulte jeune (de 15 à 45 ans) sur 250 et un sur 640 aura survécu à un cancer traité pendant l'enfance.

L'amélioration du diagnostic et du pronostic des tumeurs pédiatriques est liée à l'intensification des traitements, souvent au prix d'une augmentation de la morbidité à long terme, notamment au niveau de la fonction ovarienne (Nieman et al., 2006). Ce gain, en termes de survie s'accompagne d'une augmentation des séquelles, notamment sur le plan de la fertilité avec une destruction quasi constante de la fonction ovarienne chez les filles. Les techniques de préservation de la fertilité, notamment la cryoconservation peuvent ainsi être envisagées en dehors du contexte oncologique. C'est le cas par exemple des drépanocytoses ou des thalassémies, dont le traitement peut nécessiter une greffe de moelle, ou des tumeurs bénignes bilatérales de l'ovaire ou encore de certaines anomalies génétiques associées à une insuffisance ovarienne primaire comme le syndrome de Turner (Zacharin, 2010).

\section{Gonadotoxicité des traitements}

Les altérations des fonctions gonadiques, qu'elles soient ovariennes ou testiculaires peuvent être dues à la chimiothérapie et/ou à la radiothérapie par irradiation directe des gonades ou de l'axe hypothalamohypophysaire. Le risque d'infertilité dépend non seulement du type de tumeur et de son traitement mais aussi du sexe du patient, voire de son âge car les effets de la chimiothérapie et de la radiothérapie se manifestent différemment chez la fille et le garçon. De plus, ces effets secondaires ne se limitent pas seulement à la stérilité mais peuvent compromettre la sécrétion des stéroïdes, la mise à la place de la puberté ou bien induire une ménopause précoce (Schmidt et al., 2010).

\section{Chimiothérapie}

La chimiothérapie est responsable de lésions de l'ADN directement sur l'ovaire (Meirow \& Nugent, 2001). Ces lésions induisent une apoptose des follicules en croissance mais aussi du stock de follicules primordiaux (structures quiescentes représentant la réserve folliculaire de l'ovaire).

La toxicité de la chimiothérapie varie en fonction de la classe thérapeutique des agents utilisés, les ovaires étant extrêmement sensibles à ces traitements cytotoxiques et tout particulièrement aux agents alkylants. L'action de ces derniers produit des liaisons covalentes entres les brins d'ADN. Le clivage des deux brins d'ADN pour la réplication devient alors impossible et aboutit à un blocage de la division cellulaire. Dans la classe des alkylants, les moutardes azotées (chlorambucil), le cyclophosphamide, l'ifosfamide, le melphalan et le busulfan sont les plus toxiques pour l'ovaire, par rapport aux sels de platine (cisplatine) et aux dérivés nitrosurés (Chemaitilly et al., 2006). À cette 
toxicité propre, et au sein d'une même classe de médicaments, la toxicité dépend de la dose et de l'âge du patient et aussi des associations thérapeutiques (par exemple, association à une radiothérapie). Le conditionnement avant les greffes de cellules souches hématopoïétiques (c'est-à-dire le traitement réalisé dans un but myéloablatif de destruction des cellules malignes et en cas d'allogreffe dans le but de diminuer les épisodes de rejet) induit une toxicité ovarienne majeure quand il combine des doses élevées de busulfan et de cyclophosphamide ou de melphalan (Bakker et al., 2004). L'efficacité anti-tumorale de ces différents traitements est telle que ces protocoles sont largement utilisés chez l'enfant. Les autres classes d'agents utilisés en chimiothérapie agissent en général sur des cellules en division et sont donc moins toxiques sur les très petits follicules constituant la réserve ovarienne.

Le pool de follicules primordiaux est déterminé à la naissance chez la femme. Chez un embryon de 18 semaines, on estime qu'il existe environ six millions de follicules, alors qu'à la naissance il ne reste plus qu'un million de follicules par ovaire. Cette réserve va progressivement décroître pour atteindre 300000 à la puberté. $\mathrm{Au}$ sein de ces follicules présents après la puberté, seuls 400 vont maturer jusqu'à l'ovulation. Donc, toute lésion iatrogène (chimio ou radiothérapie) pendant la période pré-pubertaire peut induire une atteinte plus ou moins totale des fonctions ovariennes, avec un risque élevé d'insuffisance ovarienne primaire (IOP) ou d'insuffisance ovarienne aiguë. L'IOP est définie par la survenue précoce de l'épuisement de la fonction ovarienne, associant une aménorrhée et une élévation des taux de FSH supérieurs à $30 \mathrm{UI} / \mathrm{L}$, mais on considère qu'il persiste une chance de grossesse spontanée dans 5 à $6 \%$ des cas. Par exemple, une femme de plus de 25 ans recevant une poly-chimiothérapie ou une greffe de cellules souches hématopoïétiques a un risque d'IOP compris entre 80 et $100 \%$, alors que la même poly-chimiothérapie reçue avant l'âge de 20 ans permet une éventuelle grossesse spontanée de l'ordre de 28 \% (Donnez et al., 2010). L'insuffisance ovarienne aiguë est définie par une perte des fonctions ovariennes dans les cinq ans après le diagnostic de tumeur et touche particulièrement les survivantes de cancer traitées pendant l'enfance ou l'adolescence. Elle a la particularité, contrairement à l'IOP, d'être, dans certains cas, réversible.

Le risque de ces insuffisances ovariennes (Bakker et al., 2004 ; Donnez et al., 2010) après chimiothérapie est multiplié par un facteur allant de 2 à 9 par rapport à une population témoin. Selon les études, le pourcentage d'insuffisance aiguë, dans une population traitée pendant l'enfance, varie de $6 \%$ à $12 \%$ (Bakker et al., 2004), les différences étant liées au type de tumeurs.

Chez les petites filles traitées pour un cancer avant la puberté, outre le risque de stérilité, la survenue de la puberté est souvent compromise et nécessite alors une hormonothérapie substitutive au long cours. Il existe peu ou pas de publications rapportant les risques concernant la puberté après traitement à visée oncologique, et ce risque semble être maximal après conditionnement pour une greffe de cellules souches hématopoïétiques. Les risques à long terme d'un traitement substitutif prolongé et débuté précocement dans la vie sont mal connus, en particulier en ce qui concerne le cancer du sein mais également les tumeurs secondaires, notamment après traitement pour un lymphome de Hodgkin. Ce risque oncologique est à moduler en fonction de la tumeur primitive, du traitement reçu ou d'éventuelles prédispositions génétiques.

\section{Radiothérapie}

La gonadotoxicité de la radiothérapie est liée à l'action des radiations ionisantes au niveau des cellules, qu'elles soient en division ou en quiescence comme c'est le cas des follicules primordiaux. Comme pour la chimiothérapie, le degré de toxicité ovarienne dépend de l'âge au moment du traitement, du site d'irradiation, de la dose par séance et de la dose cumulée. Une dose de 5 à 20 Gy chez l'enfant (4 à 6 chez l'adulte) entraîne une atteinte irréversible de la fonction ovarienne (Wallace et al., 2003 ; Chemaitilly et al., 2006). Un conditionnement avant greffe de cellules souches hématopoïétiques avec une irradiation corporelle totale nécessite une dose de 14,4 grays et induit une IOP dans $90 \%$ des cas, si elle est pratiquée après l'âge de 10 ans (Bakker et al., 2004). De plus, les femmes ayant reçu une irradiation incluant l'utérus durant l'enfance ont un risque accru de fausses couches et de donner naissance à des enfants prématurés ou de petits poids (Signorello et al., 2006).

La toxicité de ces traitements chez la fille est donc importante alors que chez le garçon, les dommages testiculaires sont souvent moins sévères avec une puberté spontanée (grâce à des lésions moindres au niveau des cellules de Leydig, qui résistent jusqu'à une dose de $20 \mathrm{~Gy}$ ) mais la prévalence d'oligo-azoospermie à long terme (par lésion des cellules germinales, pour une dose de seulement 1,2 Gy) est aussi fréquente que les lésions ovariennes.

En résumé, les patientes ayant des risques élevés d'altération des fonctions gonadiques sont celles 
traitées par des chimiothérapies à haute dose, comportant des alkylants, par une radiothérapie pelvienne ou abdominale au-delà de 10 Gy ou par une greffe de cellules souches hématopoïétiques.

\section{Préservation des fonctions gonadiques et du tractus génital pendant le traitement}

Chez les filles, plusieurs options ont été développées pour préserver la fonction ovarienne, médicamenteuse ou chirurgicale.

Chirurgicalement, avant les radiothérapies pelviennes, on peut envisager une transposition ovarienne hors du champ d'irradiation, ce qui diminue les effets des radiations mais accroît les causes anatomiques de stérilité en augmentant la distance entre l'ovaire et la trompe. La dose de radiations reçue par les ovaires est diminuée de 5 à $10 \%$ après transposition et permet de préserver la fonction endocrine dans approximativement $60 \%$ des cas. En revanche, une étude récente (Wo \& Viswanathan, 2009) montre que 50 à $90 \%$ des femmes présentent une insuffisance ovarienne, malgré la transposition après une irradiation pelvienne. De plus, des complications de cette procédure ont été décrites (douleur chronique, lésion vasculaire, ischémie de la trompe), même si elles sont rares.

Il est aussi possible de préserver la fertilité en privilégiant la chirurgie conservatrice dans les tumeurs germinales de l'ovaire (annexectomie unilatérale préservant l'utérus et l'annexe controlatérale) et en évitant toute chirurgie extensive et radiothérapie externe dans certaines atteintes malignes de la filière génitale (rhabdomyosarcome, par exemple).

Médicalement, il a été également proposé d'utiliser soit une contraception orale pendant le traitement (chez des patientes pubères) ou des agonistes de la GnRH (Blumenfeld et al., 1996) ce qui induit une inhibition réversible de la sécrétion de la $\mathrm{GnRH}$, permettant d'arrêter la croissance folliculaire. Ce type de traitement n'apporte aucun bénéfice sur la toxicité directe de la radiothérapie ou des alkylants sur les follicules primordiaux. Cette option peut être proposée chez l'adolescente mais aucune étude clinique n'a montré de réels bénéfices (Schmidt et al., 2010).

Chez les garçons, la seule méthode de préservation de la fertilité est le déplacement temporaire de la gonade hors du champ d'irradiation, soit dans la cuisse soit dans la partie antérieure de l'abdomen.

\section{Préservation de la fertilité avant le traitement}

\section{Cryoconservation testiculaire}

Chez le garçon, la congélation de sperme est la méthode la plus répandue et la plus efficace pour préserver la fertilité. Cependant, cette technique n'est pas utilisable chez le jeune garçon, de même que le recueil par ponction testiculaire. La congélation de parenchyme testiculaire est donc la seule option à considérer chez le garçon non pubère.

Selon des travaux récents chez l'homme (Keros et al., 2007) ou expérimentaux (Milazzo et al., 2008), il semble que la cryoconservation de parenchyme testiculaire permette de maintenir un tissu testiculaire immature d'architecture normale mais aussi des cellules viables également pour leurs fonctions endocrines. Ces résultats préliminaires semblent prometteurs pour les garçons prépubères devant recevoir un traitement gonadotoxique.

En ce qui concerne l'assistance médicale à la procréation, plusieurs alternatives existent chez la femme adulte :

- La congélation embryonnaire : c'est la technique de choix. Or, elle suppose que la patiente soit en âge de procréer, le taux de grossesses est de 20 à $30 \%$ par transfert de 2 ou 3 embryons.

- La cryoconservation d'ovocytes matures : les taux de survie des ovocytes rapportés avec ces techniques sont de l'ordre de $82 \%$ alors que les taux de succès en termes de naissance ne sont que de $2 \%$.

Cependant, le recueil d'ovocytes matures nécessite une hyperstimulation ovarienne et le délai nécessaire à ce recueil est souvent incompatible avec l'urgence du traitement anti-tumoral.

Ces deux techniques de congélation ne sont pas concevables chez des enfants non pubères et la cryoconservation de cortex ovarien reste la seule technique possible car elle ne nécessite pas de stimulation et permet la conservation de cellules immatures (Weintraub et al., 2007).

\section{Cryoconservation ovarienne : résultats cliniques à partir d'ovaires pubères}

Le but essentiel de cette stratégie est la réimplantation de tissu ovarien cortical dans la cavité pelvienne (site orthotopique) ou en position sous-cutanée dans le bras (site hétérotopique) après la fin du traitement et seulement lorsque la patiente est totalement guérie. À ce jour, quinze naissances ont été obtenues après 
cryoconservation et réimplantation orthotopique de cortex provenant d'un ovaire adulte (Donnez et al., 2004 ; Siegel-Itzkovich, 2005 ; Demeestere et al., 2007 ; Meirow et al., 2007 ; Silber et al., 2008 ; Donnez \& Dolmans, 2011 ; Donnez et al., 2011) et six grossesses après réimplantation de cortex ovarien frais, entre des jumelles homozygotes (Andersen et al., 2008). Début 2009, on comptait 43 greffes réalisées avec du tissu ovarien frais ou cryoconservé, avec une restauration de cycles spontanés pendant plusieurs mois dans la majorité des cas (Demeestere et al., 2009).

C'est en 2004 que l'équipe de Donnez à Bruxelles publie la première naissance après réimplantation de cortex congelé. Il s'agit d'une jeune femme de 25 ans traitée en 1997 pour un lymphome de Hodgkin de stade IV. Avant tout traitement, un prélèvement par colioscopie de 5 fragments ovariens, qui ont été cryoconservés, a été réalisé. Le traitement du lymphome de Hodgkin a comporté une association de chimiothérapie à base d'alkylants (MOPP/ABV) et de radiothérapie (38 Gy). Onze mois après la greffe, le dosage de $\beta$ hCG et l'échographie confirmaient la grossesse permettant la naissance d'une petite fille, à terme. La publication de cette première naissance a suscité une vive polémique en raison de la spontanéité de la grossesse après ce type de traitement (Oktay \& Tilly, 2004), des risques malformatifs ou de récidive tumorale, alors qu'il existe des choix alternatifs comme les ponctions d'ovocytes.

La deuxième grossesse a été publiée l'année suivante par l'équipe israélienne de Meirow. La patiente était traitée pour un lymphome non-Hodgkinien, la congélation de fragments d'ovaire a eu lieu après une première ligne de chimiothérapie et la réimplantation a été effectuée deux ans plus tard en réinsérant les fragments de cortex au sein de l'ovaire restant. La reprise des cycles a eu lieu huit mois plus tard, puis après ponction d'ovocytes et ICSI l'obtention de la grossesse a permis la mise au monde d'une petite fille.

La troisième naissance a été obtenue après greffe de cortex ovarien congelé, en position ortho et hétérotopique, chez une jeune femme traitée par chimiothérapie pour un lymphome de Hodgkin (Demeestere et al., 2007). En outre, une équipe danoise (Silber et al., 2008) a rapporté en 2008 deux naissances après auto-transplantation en position orthotopique de greffons congelés et fécondation in vitro.

\section{Cryoconservation ovarienne : données actuelles}

En France, la cryoconservation de cortex ovarien est réalisée depuis 1995 chez l'adulte et 1998 chez l'enfant (Poirot et al., 2007). Dans le fichier national du GRECOT
(Groupe de Recherche et d'Étude sur la Cryoconservation Ovarienne et Testiculaire), 436 cryoconservations étaient colligées au début 2007. L'âge moyen au moment du prélèvement était de $17,6 \pm 9$ ans, et $21 \%$ des patientes avaient moins de 12 ans. La cryoconservation était majoritairement proposée dans des cas de pathologies hématologiques malignes (60\%), de tumeurs solides (majoritairement à l'âge pédiatrique) et de pathologies bénignes dans $9 \%$ des cas (drépanocytose, maladies de système, thalassémies...). L'étude histologique des greffons au moment de la congélation a permis de montrer une localisation tumorale secondaire dans deux cas (leucémie et lymphome).

Historiquement, en France, la congélation ovarienne a d'abord été réalisée dans le cadre d'un CCPPRB (Comité Consultatif de Protection des Personnes dans la Recherche Biomédicale), dont le promoteur était le Pr. Piver à Limoges. Puis, son cadre législatif a fait partie de la loi relative à la Bioéthique du 6 août 2004 et de l'article L 2141-11 du Code de la Santé Publique. Ce texte autorise une autoconservation du tissu gonadique dès lors qu'un traitement altérant la fertilité est envisagé ou que la fertilité risque d'être altérée pour des causes endogènes. Donc, la conservation, à des fins autologues du tissu ovarien (c'est-à-dire ne pouvant être utilisée qu'au bénéfice de la patiente donneuse), peut aujourd'hui être considérée comme une pratique relevant du soin, sous contrôle de l'Agence de la Biomédecine, et non plus de la recherche. Son utilisation est en revanche plus sujette à discussion car la finalité attribuée par la loi à la cryoconservation ovarienne est son utilisation au titre d'une assistance médicale à la procréation (AMP). Or, l'autogreffe en position orthotopique ne peut pas être considérée comme une AMP. Pour l'instant, on considère donc que la réimplantation doit entrer dans un projet de recherche biomédicale.

Une naissance après greffe de cortex ovarien congelé chez une femme adulte a été rapportée en 2009, par les équipes de Besançon et Limoges (Roux et al., 2010).

\section{Cryoconservation ovarienne à partir d'ovaires non pubères}

Contrairement aux publications sur les naissances à partir d'ovaires congelés adultes et l'expérience de congélation d'ovaires non pubères (France ou Israël), aucune donnée n'est disponible chez la femme ou même chez l'animal sur le devenir de ces ovaires conservés avant la puberté (immatures). Pour analyser les fonctions ovariennes endocrines et exocrines, après 
réimplantation de ces greffons immatures, nous avons mis au point un modèle de greffe orthotopique non vascularisée chez la souris et la brebis.

\section{Stratégie de recherche et résultats}

\section{Modèle souris}

Des greffes ovariennes ont été réalisées chez des souris de fond génétique $129 \mathrm{~Sv} / \mathrm{J}$. Les ovaires définis comme immatures provenaient de souris non pubères, opérées avant l'âge de 3 semaines. Les souris receveuses étaient soit considérées comme immatures (3 semaines) soit adultes (2-3 mois). Les ovaires ont été enlevés chez toutes ces souris avant la greffe, pour s'assurer que les sécrétions hormonales et les gestations provenaient bien $\mathrm{du}$ greffon. $\mathrm{Au}$ total, 86 femelles ont eu une transplantation ovarienne en position orthotopique. Après la greffe, la restauration d'une activité œstrale cyclique, étudiée par frottis vaginal quotidien, était considérée comme le signe d'une activité hormonale provenant d'un greffon fonctionnel. La reprise d'une activité hormonale cyclique est intervenue en moyenne après $14,9 \pm 2,8$ jours après la greffe.

L'étude histologique des greffons ovariens un mois après la greffe montre qu'il existe au sein de ces ovaires des follicules à tous les stades du développement et l'étude immunohistochimique confirme la présence d'une néovascularisation ainsi qu'une prolifération cellulaire active (marquage PCNA et Ki67). En revanche, la perte folliculaire est de l'ordre de $50 \%$ après greffe, clairement liée à l'ischémie ovarienne secondaire à l'ovariectomie et à la greffe, alors que la cryoconservation ou l'âge du receveur n'avaient pas d'influence.

Après accouplement, 25 à $50 \%$ des femelles greffées ont mis bas, après des gestations de durée normale. Le nombre de gestations par souris greffée était compris entre 1 et 3 . La dernière gestation est apparue entre 3 et 9 mois après la greffe, suggérant un épuisement rapide des greffons, ce qui a été confirmé histologiquement (Sauvat et al., 2008).

\section{Modèle brebis}

Ces résultats encourageants obtenus chez la souris ont conduit à réaliser une étude similaire chez la brebis. Le choix de la brebis se justifie par son caractère monoovulant et sa physiologie proche de celle de la femme.
Douze brebis pré-Alpes du Sud ont été opérées et suivies pendant plus de 3 ans. Nous avons mis au point un modèle de réimplantation ovarienne orthotopique, non vascularisée, en réimplantant chez le même animal des ovaires non pubères soit frais, soit après congélation avant ou après la puberté. Dans ce groupe, un ovaire était enlevé et congelé avant la puberté, la brebis faisait sa puberté grâce à l'ovaire controlatéral qui était à son tour enlevé au moment de la greffe.

Chez ces douze animaux, des dosages hormonaux mensuels (progestérone, œestradiol et FSH) ont montré une sécrétion hormonale dans les trois mois après la greffe, correspondant à l'initiation et au maintien d'une puberté normale, à la date physiologique de la puberté. Après 39 à 41 mois d'étude, il n'existait pas de signe en faveur d'un épuisement du greffon (taux de FSH normaux), ce que confirmait également la présence de follicules viables en histologie.

Après mise en accouplement, lors de la première saison sexuelle, six brebis étaient gestantes, réparties dans les trois groupes et six petits sont nés. Au total sur les trois saisons d'activité sexuelle étudiées, 15 naissances ont été obtenues, chez six brebis greffées. Les nouveaux-nés ne présentaient aucune malformation évidente à l'examen macroscopique ou dans certains cas sur des biopsies multiples.

\section{Cryoconservation gonadique et marques épigénétiques}

Si la cryoconservation offre une perspective optimiste aux enfants après un traitement oncologique, ces techniques soulèvent des questions médicales, voire éthiques.

Des anomalies du développement ont été rapportées à plusieurs reprises chez les enfants issus de techniques aussi variées que la stimulation ovarienne, la fécondation in vitro ou les injections de spermatozoïdes intra-cytoplasmiques. Les anomalies épigénétiques dans la descendance après des techniques d'assistance médicale à la procréation n'ont jamais été rapportées chez la souris, alors que chez la brebis le Large Offspring Syndrome, équivalent du syndrome de WiedemannBeckwith chez l'Homme, a été décrit. L'empreinte parentale est un mécanisme de régulation génique responsable de la non-équivalence des deux génomes parentaux. L'activité fonctionnelle d'un gène soumis à empreinte parentale dépend de son origine parentale et son expression sera alors monoallélique. Ce risque d'anomalies est d'autant plus important à prendre en compte lorsque le processus de conservation est une 
manipulation supplémentaire. Les ovaires prélevés avant la puberté (avant la mise en place de l'empreinte au niveau de l'ovocyte) sont aussi plus sensibles à toute intervention externe.

Pour évaluer ce risque, nous avons étudié, dans nos modèles expérimentaux, l'état de méthylation de gènes soumis à empreinte tels que $H 19$ et $K v D M R$ (impliqués dans le syndrome de Wiedemann-Beckwith) dans le muscle, la langue et le rein des descendants des souris greffées par rapport à celui de souris sauvages de même âge.

Les résultats n'ont pas montré de différence statistiquement significative par rapport à une population témoin de même âge. Cette conclusion est encourageante, cependant on ne doit pas perdre de vue les points suivants: la faible taille de l'échantillon testée, le peu de données disponibles chez la souris sur le profil de méthylation de la DMR (Differentially Methylated Region) du gène $H 19$ et notamment son évolution en fonction de l'âge et du tissu. Nos résultats ne montrent pas non plus de différence dans le statut de méthylation de $K v D M R$ entre les souriceaux dont la mère avait été greffée et les souriceaux sauvages (Sauvat et al., 2008). Là encore, ces résultats sont d'interprétation difficile du fait de la petite taille des effectifs.

Chez la brebis, nous avons comparé les profils de méthylation de la région du récepteur de l'IGF2 (Igf2r) des couples mères greffées et descendants (en comparant le nombre de cytosines méthylées au sein des dinucléotides CpG sur les 15 dinucléotides présents dans la séquence par rapport au nombre total de dinucléotides analysables). Les résultats ne montrent pas de différence significative mais leur interprétation nécessite la prudence à cause de la faiblesse des effectifs.

L'autre controverse concernant la greffe de tissu congelé est le risque de réintroduire des cellules malignes au moment de la greffe, notamment quand la tumeur primitive peut donner des métastases de localisation gonadique, comme les leucémies ou les lymphomes (Shaw et al., 1996 ; Meirow et al., 2008). La croissance complète in vitro et la maturation des ovocytes ne peut être possible qu'après une manipulation complexe des étapes multiples mimant ce qui se passe in vivo dans l'ovaire. La culture in vitro de follicules primordiaux et l'isolement de ces follicules à partir d'ovaires congelés permettront probablement d'éliminer ce risque mais restent pour l'instant des techniques très expérimentales, sans application clinique immédiate.

\section{Conclusion}

Environ un tiers des jeunes femmes exposées à la chimiothérapie développent une insuffisance ovarienne ; il est donc très souhaitable de proposer une congélation de tissu ovarien à toutes les jeunes enfants, adolescentes et femmes en âge de procréer nécessitant une chimiothérapie avec des agents alkylants, ce qui constitue une menace majeure pour leur fertilité.

L'évaluation des candidats potentiels pour une préservation de la fertilité doit maintenant être systématiquement prise en compte chez des enfants traités pour une tumeur (chimiothérapie haute dose, radiothérapie englobant les gonades) ou ceux recevant une greffe de cellules souches hématopoïétiques pour une pathologie maligne ou bénigne. Parmi toutes les possibilités, la congélation de tissu ovarien semble la meilleure technique, voire la seule avant la puberté dont les résultats soient encourageants, que ce soit chez l'adulte ou dans les modèles expérimentaux. Chez le garçon, en regard des résultats préliminaires, la conservation de tissu testiculaire sera probablement également à envisager.

Deux articles très récents ouvrent la voie vers un nouveau traitement par l'imatinib (Gleevec) comme adjuvant pour protéger les ovocytes des effets délétères des agents tels que le cisplatine, en bloquant la voie apoptotique activée par cette drogue. C'est une nouvelle piste qui aura le mérite d'être testée dans un futur proche (Gonfloni et al., 2009). D'autre part, le groupe du Pr. Hsueh a manipulé les protéines PTEN et PI3kinase en utilisant respectivement des inhibiteurs et des activateurs de cette voie afin de permettre l'activation folliculaire de souriceaux nouveaux-nés. Après transplantation chez des souris hôtes ovariectomisées, les follicules traités se sont développés jusqu'au stade préovulatoire permettant l'ovulation d'œufs matures. Ces oufs matures ne présentent pas de modifications épigénétiques des gènes soumis à empreinte et ont permis la naissance de souriceaux en bonne santé ayant la capacité de se reproduire ( $\mathrm{Li}$ et al., 2010). Ces travaux ont été poursuivis chez la femme à l'aide de fragments de cortex ovarien de patientes atteintes de cancer. Après traitement par un inhibiteur de la phosphatase PTEN, et xénotransplantation sous la capsule rénale de souris immuno-déficientes, des follicules primordiaux se sont développés jusqu'au stade pré-ovulatoire avec des ovocytes capables de subir une maturation nucléaire.

Cependant l'information donnée au patient et à sa famille au sujet des résultats, après greffe de ces tissus congelés avant la puberté, doit rester prudente ; elle doit comporter à la fois les espoirs mis dans cette technique mais aussi les incertitudes, voire les risques.

\section{Références}

Andersen C.Y., Rosendahl M., Byskov A.G., Loft A., Ottosen C., Dueholm M., Schmidt K.L., Andersen A.N., Ernst E., 
Two successful pregnancies following autotransplantation of frozen/thawed ovarian tissue. Hum Reprod, 2008, $23,2266-2272$.

Bakker B., Oostdijk W., Bresters D., Walenkamp M.J., Vossen J.M., Wit J.M., Disturbances of growth and endocrine function after busulphan-based conditioning for haematopoietic stem cell transplantation during infancy and childhood. Bone Marrow Transplant, 2004, 33, 1049-1056.

Blumenfel Z., Avivi I., Linn S., Epelbaum R., Ben-Shahar M., Haim N., Prevention of irreversible chemotherapyinduced ovarian damage in young women with lymphoma by a gonadotrophin-releasing hormone agonist in parallel to chemotherapy. Hum Reprod, 1996, 11, 1620-1626.

Chemaitilly W., Mertens A.C., Mitby P., Whitton J., Stovall M., Yasui Y., Robison L.L., Sklar C.A., Acute ovarian failure in the childhood cancer survivor study. $J$ Clin Endocrinol Metab, 2006, 91, 1723-1728.

Demeestere I., Simon P., Emiliani S., Delbaere A., Englert Y., Fertility preservation: successful transplantation of cryopreserved ovarian tissue in a young patient previously treated for Hodgkin's disease. Oncologist, 2007, 12, 1437-1442.

Demeestere I., Simon P., Emiliani S., Delbaere A., Englert Y., Orthotopic and heterotopic ovarian tissue transplantation. Hum Reprod Update, 2009, 15, 649-665.

Donnez J., Dolmans M.M., Preservation of fertility in females with haematological malignancy. Br J Haematol, 2011, $154,175-184$.

Donnez J., Dolmans M.M., Demylle D., Jadoul P., Pirard C., Squifflet J., Martinez-Madrid B., van Langendonckt A., Livebirth after orthotopic transplantation of cryopreserved ovarian tissue. Lancet, 2004, 364, 1405-1410.

Donnez J., Jadoul P., Squifflet J., Van Langendonckt A., Donnez O., Van Eyck A.S., Marinescu C., Dolmans M.M., Ovarian tissue cryopreservation and transplantation in cancer patients. Best Pract Res Clin Obstet Gynaecol, 2010, 24, 87-100.

Donnez J., Silber S., Andersen C.Y., Demeestere I., Piver P., Meirow D., Pellicer A., Dolmans M.M., Children born after autotransplantation of cryopreserved ovarian tissue. A review of 13 live births. Ann Med, 2011 (sous presse).

Gonfloni S., Di Tella L., Caldarola S., Cannata S.M., Klinger F.G., Di Bartolomeo C., Mattei M., Candi E., De Felici M., Melino G., Cesarini G., Inhibition of the c-Abl-TAp63 pathway protects mouse oocytes from chemotherapyinduced death. Nat Med, 2009, 15, 1179-1185.

Keros V., Hultenby K., Borgstrom B., Fridstrom M., Jahnukainen K., Hovatta O., Methods of cryopreservation of testicular tissue with viable spermatogonia in pre-pubertal boys undergoing gonadotoxic cancer treatment. Hum Reprod, 2007, 22, 1384-1395.

Li J., Kawamura K., Cheng Y., Liu S., Klein C., Duan E.K., Hsueh A.J., Activation of dormant ovarian follicles to generate mature eggs. Proc Natl Acad Sci USA, 2010, 107, 10280-10284.
Meirow D., Nugent D., The effects of radiotherapy and chemotherapy on female reproduction. Hum Reprod Update, 2001, 7, 535-543.

Meirow D., Levron J., Eldar-Geva T., Hardan I., Fridman E., Yemini Z., Dor J., Monitoring the ovaries after autotransplantation of cryopreserved ovarian tissue: endocrine studies, in vitro fertilization cycles, and live birth. Fertil Steril, 2007, 87, 418 e7-418 e15.

Meirow D., Hardan I., Dor J., Fridman E., Elizur S., Ra'anani H., Slyusarevsky E., Amariglio N., Schiff E., Rechavi G., Nagler A., Ben Yehuda D., Searching for evidence of disease and malignant cell contamination in ovarian tissue stored from hematologic cancer patients. Hum Reprod, 2008, 23, 1007-1013.

Milazzo J.P., Vaudreuil L., Cauliez B., Gruel E., Masse L., Mousset-Siméon N., Macé B., Rives N., Comparison of conditions for cryopreservation of testicular tissue from immature mice. Hum Reprod, 2008, 23, 17-28.

Nieman C.L., Kazer R., Brannigan R.E., Zoloth L.S., Chase-Lansdale P.L., Kinahan K., Dilley K.J., Roberts D., Shea L.D., Woodruff T.K., Cancer survivors and infertility: a review of a new problem and novel answers. J Support Oncol, 2006, 4, 171-178.

Oktay K., Tilly J., Livebirth after cryopreserved ovarian tissue autotransplantation. Lancet, 2004, 364, 20912092 ; author reply 2092-2093.

Poirot C.J., Martelli H., Genestie C., Golmard J.L., Valteau-Couanet D., Hélardot P., Pacquement H., Sauvat F., Tabone M.D., Philippe-Chomette P., Espérou H., Baruchel A., Brugières L., Feasibility of ovarian tissue cryopreservation for prepubertal females with cancer. Pediatr Blood Cancer, 2007, 49, 74-78.

Roux C., Amiot C., Agnani G., Aubard Y., Rohrlich P.S., Piver P., Live birth after ovarian tissue autograft in a patient with sickle cell disease treated by allogeneic bone marrow transplantation. Fertil Steril, 2010, 93, 2413 e15e19.

Sauvat F., Capito C., Sarnacki S., Poirot C., Bachelot A., Meduri G., Dandolo L., Binart N., Immature cryopreserved ovary restores puberty and fertility in mice without alteration of epigenetic marks. PLoS One, 2008, 3, e1972.

Schmidt K.T., Larsen E.C., Andersen C.Y., Andersen A.N., Risk of ovarian failure and fertility preserving methods in girls and adolescents with a malignant disease. BJOG, 2010, 117, 163-174.

Shaw J.M., Bowles J., Koopman P., Wood E.C., Trounson A.O., Fresh and cryopreserved ovarian tissue samples from donors with lymphoma transmit the cancer to graft recipients. Hum Reprod, 1996, 11, 1668-1673.

Siegel-Itzkovich J., Woman gives birth after receiving transplant of her own ovarian tissue. BMJ, 2005, 331, 70 .

Signorello L.B., Cohen S.S., Bosetti C., Stovall M., Kasper C.E., Weathers R.E., Whitton J.A., Green D.M., 
Donaldson S.S., Mertens A.C., Robison L.L., Boice J.D., Jr., Female survivors of childhood cancer: preterm birth and low birth weight among their children. J Natl Cancer Inst, 2006, 98, 1453-1461.

Silber S.J., DeRosa M., Pineda J., Lenahan K., Grenia D., Gorman K., Gosden R.G., A series of monozygotic twins discordant for ovarian failure: ovary transplantation (cortical versus microvascular) and cryopreservation. Hum Reprod, 2008, 23, 1531-1537.

Skinner R., Wallace W.H., Levitt G.A., Long-term follow-up of people who have survived cancer during childhood. Lancet Oncol, 2006, 7, 489-498.

Stiller C.A., Desandes E., Danon S.E., Izarzugaza I., Ratiu A., Vassileva-Valerianova Z., Steliarova-Foucher E., Cancer incidence and survival in European adolescents (1978-1997). Report from the Automated Childhood
Cancer Information System Project. Eur J Cancer, 2006, 42, 2006-2018.

Wallace W.H., Thomson A.B., Kelsey T.W., The radiosensitivity of the human oocyte. Hum Reprod, 2003, $18,117-121$.

Weintraub M., Gross E., Kadari A., Ravitsky V., Safran A., Laufer N., Revel A., Should ovarian cryopreservation be offered to girls with cancer? Pediatr Blood Cancer, 2007, 48, 4-9.

Wo J.Y., Viswanathan A.N., Impact of radiotherapy on fertility, pregnancy, and neonatal outcomes in female cancer patients. Int J Radiat Oncol Biol Phys, 2009, 73, 1304-1312.

Zacharin M., Disorders of ovarian function in childhood and adolescence: evolving needs of the growing child. An endocrine perspective. BJOG, 2010, 117, 156-162. 